\title{
DÉCOMPOSITION DE LA COHOMOLOGIE CYCLIQUE BIVARIANTE DES ALGÈBRES COMMUTATIVES
}

\author{
PHILIPPE NUSS
}

\section{Introduction.}

Dans [9], Jones et Kassel ont associé à tout couple $(A, B)$ d'algèbres sur un anneau commutatif $k$ un $k$-module Z-gradué - la cohomologie cyclique bivariante de $(A, B) \mathrm{HC}^{*}(A, B)$ - qui possède les propriétés suivantes:

(i) $\mathrm{HC}^{*}(A, B)$ est un bifoncteur contravariant en $A$ et covariant en $B$.

(ii) Il existe des isomorphismes naturels:

$$
\mathrm{HC}^{*}(k, A) \cong \mathrm{HC}_{-*}^{-}(A) \text { et } \mathrm{HC}^{*}(A, k) \cong \mathrm{HC}^{*}(A)
$$

où $\mathrm{HC}^{*}(A)$ est la cohomologie cyclique de Connes [3] et $\mathrm{HC}_{*}^{-}$est l'homologie cyclique négative de Goodwillie [7].

(iii) Il existe un produit gradué naturel:

$$
\mathrm{HC}^{*}\left(A_{1}, B_{1} \otimes C\right) \otimes \mathrm{HC}^{*}\left(C \otimes A_{2}, B_{2}\right) \rightarrow \mathrm{HC}^{*}\left(A_{1} \otimes A_{2}, B_{1} \otimes B_{2}\right) .
$$

Ce produit permet de faire opérer la cohomologie cyclique bivariante sur les groupes de (co)homologie cyclique (négative, périodique) de manière compatible avec les longues suites exactes de Connes.

(iv) Il existe une longue suite exacte:

$$
\ldots \stackrel{B}{\longrightarrow} \mathrm{HC}^{n-2}(A, B) \stackrel{S}{\longrightarrow} \mathrm{HC}^{n}(A, B) \stackrel{I}{\rightarrow} \mathrm{HH}^{n}(A, B) \stackrel{B}{\longrightarrow} \mathrm{HC}^{n-1}(A, B) \stackrel{s}{\rightarrow} \ldots
$$

étendant les suites exactes de Connes.

On trouvera d'autres propriétés de la cohomologie cyclique bivariante dans [11] [12].

Le premier but de cet article est de montrer que lorsque $k$ contient le corps $Q$ des nombres rationnels et que $A$ et $B$ sont des algèbres commutatives, alors la cohomologie cyclique bivariante a une décomposition naturelle de "type Hodge":

$$
\mathrm{HC}^{*}(A, B)=\prod_{i \in \mathbf{Z}} \mathrm{HC}_{(i)}^{*}(A, B)
$$

Reçu le 3 Décembre 1990. 
généralisant celles de [2][4][15][17][20] en (co)homologie cyclique. On montre aussi que cette décomposition est compatible au produit (iii) ci-dessus, ce qui, pour tout couple $(i, j)$ d'entiers, induit une application:

$$
\mathrm{HC}_{(i)}^{*}\left(A_{1}, B_{1} \otimes C\right) \otimes \mathrm{HC}_{(j)}^{*}\left(C \otimes A_{2}, B_{2}\right) \rightarrow \mathrm{HC}_{(i+j)}^{*}\left(A_{1} \otimes A_{2}, B_{1} \otimes B_{2}\right) .
$$

De même la longue suite exacte de (iv) est somme directe de longues suites exactes de la forme:

$$
\ldots \stackrel{B}{\longrightarrow} \mathrm{HC}_{(i+1)}^{n-2}(A, B) \stackrel{s}{\longrightarrow} \mathrm{HC}_{(i)}^{n}(A, B) \stackrel{B}{\longrightarrow} \mathrm{HC}_{(i+1)}^{n-1}(A, B) \stackrel{s}{\longrightarrow} \ldots
$$

Par ailleurs, Loday [15] a montré que la décomposition de l'homologie cyclique d'une algèbre commutative $A$ provenait d'opérations $\psi^{l}$ qu'il construit sur le $(b, B)$-bicomplexe $\mathscr{B}(A)$. Une question naturelle est de savoir si ces opérations sont induites par de éléments de $\operatorname{HC}^{0}(A, A)$. La réponse est positive bien que techniquement non triviale. En effet, les endomorphismes $\psi^{l}$ de $\mathscr{B}(A)$ ne commutent pas à $S$ comme ils le devraient pour donner lieu à des cocycles bivariants, mais vérifient la relation:

$$
S \psi^{l}=l \psi^{l} S .
$$

Pour tourner la difficulté, nous définissons pour chaque élément $l$ de l'anneau de base $k$, une variante $\mathrm{HC}^{*}(A, B)(l)$ de la cohomologie cyclique bivariante, basée sur les applications $f$ entre $(b, B)$-bicomplexes vérifiant: $S f=l f S$. Pour $A$ commutative, l'operation $\psi^{l}$ apparaît alors comme un élément de $\operatorname{HC}^{0}(A, A)(l)$. Nous donnons ensuite un théorème des coefficients universels et nous montrons que, lorsque $k$ est un corps, alors $\mathrm{HC}^{*}(A, B)(l)$ est isomorphe (non canoniquement) à $\operatorname{HC}^{*}(A, B)$.

On en déduit une structure de $\gamma$-anneau au sens de [1] sur la cohomologie cyclique bivariante.

\section{Décomposition de l'homologie cyclique négative.}

Comme la cohomologie cyclique bivariante contient comme cas particuliers la cohomologie cyclique et l'homologie cyclique négative, il convient, avant de décomposer la cohomologie cyclique bivariante, de décomposer les groupes $\mathrm{HC}^{*}(A)$ et $\mathrm{HC}_{*}^{-}(A)$. Pour $\mathrm{HC}^{*}(A)$, cela a été fait par Natsume-Schack [17]. Pour l'homologie cyclique négative, c'est nouveau.

Auparavant, rappelons les notations. On fixe un anneau commutatif $k$. Dans la suite, toutes les algèbres sont supposées associatives et unitaires.

Pour toute $k$-algèbre $A$, nous notons $\left(C_{*}(A), b\right)$ le complexe normalisé de Hochschild donné par

$$
C_{n}(A)=A \otimes \bar{A}^{\otimes n}
$$


où les produits tensoriels sont pris au-dessus de $k$ et où $b\left(a_{0} \otimes \ldots \otimes a_{n}\right)=\sum_{i=0}^{n-1}(-1)^{i} a_{0} \otimes \ldots \otimes a_{i} a_{i+1} \otimes \ldots \otimes a_{n}+(-1)^{n} a_{n} a_{0} \otimes a_{1} \otimes \ldots \otimes a_{n-1}$.

Ici on a posé $\bar{A}=\operatorname{coker}(k \rightarrow A)$. L'opérateur $B$ de Connes, donné par

$$
B\left(a_{0} \otimes \ldots \otimes a_{n}\right)=\sum_{i=0}^{n}(-1)^{n i} 1 \otimes a_{i} \otimes \ldots \otimes a_{n} \otimes a_{0} \otimes \ldots \otimes a_{i-1}
$$

permet de définir le bicomplexe $\mathscr{B}(A)$ (dénoté par $\mathscr{B}(A)_{\text {norm }}$ dans [16])

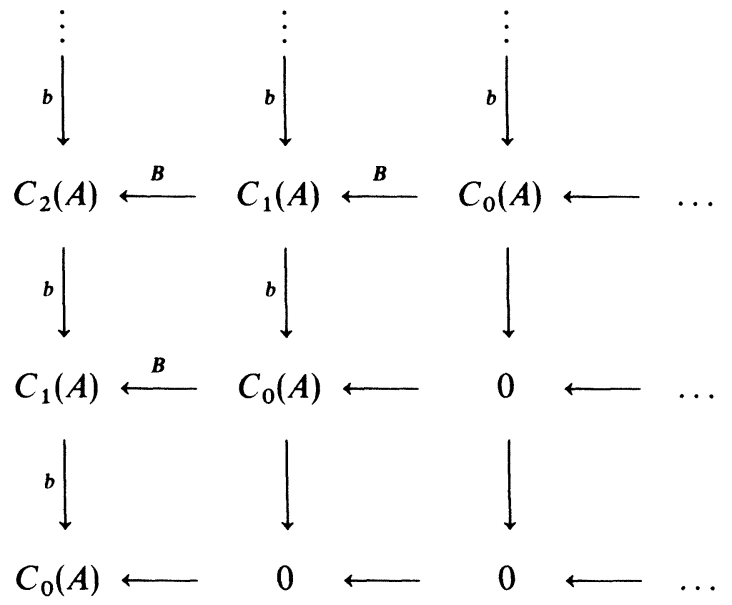

On a la suite exacte de complexes

$$
0 \longrightarrow C_{*}(A) \stackrel{I}{\rightarrow} \mathscr{B}(A) \stackrel{s}{\rightarrow} \mathscr{B}(A)[2] \longrightarrow 0 .
$$

Si l'anneau $k$ contient le corps des nombres rationnels et si $A$ est une $k$-algèbre commutative, Gerstenhaber et Schack [5] ont montré que le complexe de Hochschild a une décomposition naturelle en somme directe de sous-complexes

$$
C_{*}(A)=\bigoplus_{i \geqq 0} C_{*}^{(i)}(A) \text {. }
$$

En passant à l'homologie, on a une décomposition naturelle de l'homologie de Hochschild de $A$ qui coïncide avec celle du théorème 8.6 de Quillen [18]:

$$
\mathrm{HH}_{*}(A)=\bigoplus_{i \geqq 0} \mathrm{HH}_{*}^{(i)}(A) .
$$

Rappelons que $\mathrm{HH}_{*}^{(1)}(A)$ est l'homologie de Harrison [8] [18] [19] de $A$. Pour ce qui est de l'homologie cyclique, Loday [15] et Natsume-Schack [17] ont 
vérifié que l'opérateur $B$ envoie $C_{*}^{(i)}(A)$ dans $C_{*}^{(i+1)}(A)$, ce qui permet de décomposer $\mathscr{B}(A)$ comme somme directe

$$
\mathscr{B}(A)=\bigoplus_{i \geqq 0} \mathscr{B}^{(i)}(A)
$$

de sous-complexes $\mathscr{B}^{(i)}(A)$ :

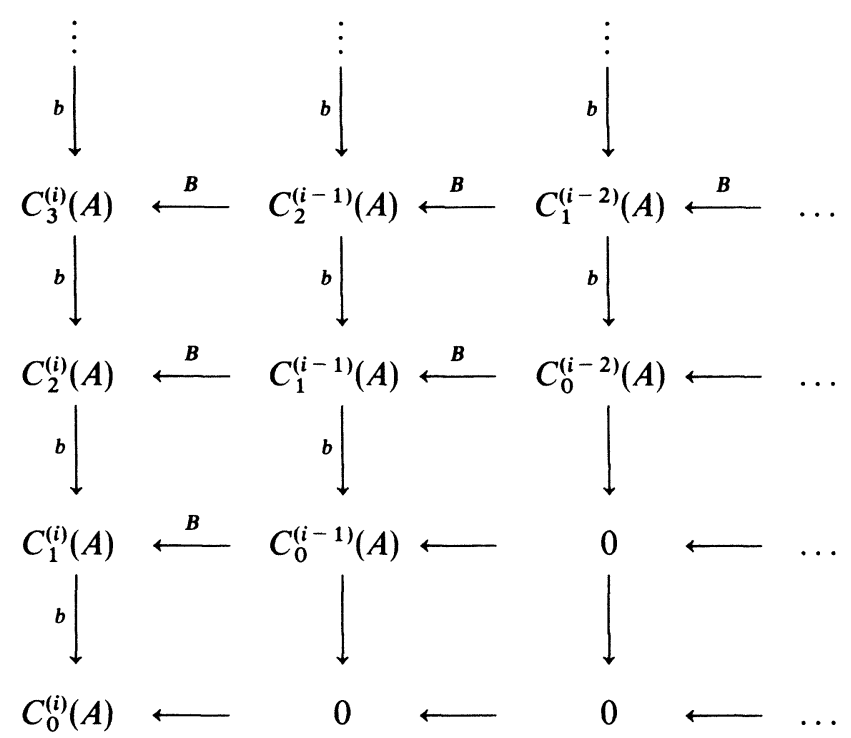

La suite exacte de complexes (1.1) reliant $C_{*}(A)$ et $\mathscr{B}(A)$ est la somme directe de suites exactes de la forme

$$
0 \longrightarrow C_{*}^{(i)}(A) \stackrel{I}{\rightarrow} \mathscr{B}^{(i)}(A) \stackrel{S}{\rightarrow} \mathscr{B}^{(i-1)}(A)[2] \longrightarrow 0 .
$$

On sait que la cohomologie cyclique $\mathrm{HC}^{*}(A)$ est défine par

$$
\mathrm{HC}^{*}(A)=H^{*}(\operatorname{Hom}(\mathscr{B}(A), k))
$$

ce qui donne:

$$
\mathrm{HC}^{*}(A)=\prod_{i \geqq 0} \mathrm{HC}_{(i)}^{*}(A)
$$

où on a posé:

$$
\mathrm{HC}_{(i)}^{*}(A)=H^{*}\left(\operatorname{Hom}\left(\mathscr{B}^{(i)}(A), k\right)\right) .
$$

Ces groupes sont liés à ceux de [17] par la relation

$$
\operatorname{HC}_{(i)}^{n}(A)=H_{\lambda}^{i, n-i}(A)
$$


Pour l'homologie cyclique négative, définissons le complexe $\mathscr{B}^{-(i)}(A)$ par

$$
\mathscr{B}_{n}^{-(i)}(A)=\prod_{p \geqq 0} C_{n+2 p}^{(i+p)}(A)
$$

la différentielle étant $b+B$. On note son homologie par $H C_{*}^{-(i)}(A)$. Il est immédiat que le complexe produit

$$
\prod_{i \in Z} \mathscr{B}^{-(i)}(A)
$$

est isomorphe au $(b, B)$-complexe négatif $\mathscr{B}^{-}(A)$ qui définit l'homologie cyclique négative [7]. D'où la décomposition

$$
\mathrm{HC}_{*}^{-}(A)=\prod_{i \in \mathrm{Z}} \mathrm{HC}_{*}^{-(i)}(A) \text {. }
$$

On remarquera qu'à la différence de l'homologie cyclique, la décomposition de l'homologie cyclique négative est un produit et non une somme directe et que les termes $\mathrm{HC}_{*}^{-(i)}(A)$ sont en général non nuls aussi bien pour $i$ positif que pour $i$ négatif.

\section{Décomposition de la cohomologie cyclique bivariante.}

Rappelons la définition de la cohomologie cyclique bivariante telle qu'elle est donnée dans [9].

Si $A$ et $B$ sont deux $k$-algèbres, posons

$$
\begin{aligned}
\operatorname{Hom}_{S}(\mathscr{B}(A), \mathscr{B}(B)) & =\operatorname{Ker}(\operatorname{ad}(S): \operatorname{Hom}(\mathscr{B}(A), \mathscr{B}(B)) \rightarrow \\
& \rightarrow \operatorname{Hom}(\mathscr{B}(A), \mathscr{B}(B))[2]) .
\end{aligned}
$$

Rappelons que pour deux complexes $\left(C_{*}, d\right)$ et $\left(C_{*}^{\prime}, d^{\prime}\right),\left(\operatorname{Hom}\left(C_{*}, C_{*}^{\prime}\right), \partial\right)$ désigne le complexe des applications graduées entre les complexes $C_{*}$ et $C_{*}^{\prime}$, la différentielle étant donnée par

$$
\partial(f)=d^{\prime} f-(-1)^{|f|} f d
$$

pour $f \in \operatorname{Hom}\left(C_{*}, C_{*}^{\prime}\right)$ homogène de degré $|f|$. Le morphisme de complexes $\operatorname{ad}(S)$ est défini par:

$$
\operatorname{ad}(S)(f)=S f-f S .
$$

Alors les groupes de cohomologie cyclique bivariante sont définis par

$$
\mathrm{HC}^{n}(A, B)=H_{-n}\left(\operatorname{Hom}_{S}(\mathscr{B}(A), \mathscr{B}(B))\right)
$$

et les groupes de cohomologie de Hochschild bivariante sont définis par 


$$
\mathrm{HH}^{n}(A, B)=H_{-n}\left(\operatorname{Hom}\left(C_{*}(A), C_{*}(B)\right)\right) .
$$

Énonçons le théorème principal de ce paragraphe.

THÉORÈme 2.5. Soient $A$ et $B$ deux algèbres commutatives sur un anneau commutatif $k$ contenant $Q$, alors on a des décompositions naturelles

$$
\mathrm{HC}^{*}(A, B)=\prod_{i \in \mathcal{Z}} \mathrm{HC}_{(i)}^{*}(A, B) \text { et } \mathrm{HH}^{*}(A, B)=\prod_{i \in \mathcal{Z}} \mathrm{HH}_{(i)}^{*}(A, B)
$$

telles que

1) (spécialisation) pour tout couple d'entiers $(n, i)$, on a

$$
\begin{aligned}
& \mathrm{HC}_{(i)}^{n}(A, k)=\mathrm{HC}_{(-i)}^{n}(A) \text { et } \mathrm{HC}_{(i)}^{n}(k, B)=\mathrm{HC}_{-n}^{-(i)}(B) \\
& \mathrm{HH}_{(i)}^{n}(A, k)=\mathrm{HH}_{(-i)}^{n}(A) \text { et } \mathrm{HH}_{(i)}^{n}(k, B)=\mathrm{HH}_{-n}^{(i)}(B)
\end{aligned}
$$

2) il y a une longue suite

$$
\ldots \stackrel{B}{\rightarrow} \mathrm{HC}_{(i+1)}^{n-2}(A, B) \stackrel{s}{\rightarrow} \mathrm{HC}_{(i)}^{n}(A, B) \stackrel{I}{\rightarrow} \mathrm{HH}_{(i)}^{n}(A, B) \stackrel{B}{\rightarrow} \mathrm{HC}_{(i+1)}^{n-1}(A, B) \stackrel{s}{\rightarrow} \ldots
$$

qui est exacte lorsque A est projectif comme module sur $k$.

Démonstration. Pour décomposer $\mathrm{HH}^{*}(A, B)$, il suffit de poser

$$
\operatorname{Hom}^{(i)}\left(C_{*}(A), C_{*}(B)\right)=\prod_{s-r=i} \operatorname{Hom}\left(C_{*}^{(r)}(A), C_{*}^{(s)}(B)\right)
$$

et

$$
\mathrm{HH}_{(i)}^{n}(A, B)=H_{-n}\left(\operatorname{Hom}^{(i)}\left(C_{*}(A), C_{*}(B)\right)\right) .
$$

On vérifie alors facilement que $\operatorname{Hom}\left(C_{*}(A), C_{*}(B)\right) \cong \prod_{i \in Z} \operatorname{Hom}^{(i)}\left(C_{*}(A), C_{*}(B)\right)$, ce qui donne la décomposition pour $\mathrm{HH}^{*}(A, B)$.

Passons à la cohomologie cyclique bivariante. Posons

$$
\operatorname{Hom}^{(i)}(\mathscr{B}(A), \mathscr{B}(B))=\prod_{s=r=i} \operatorname{Hom}\left(\mathscr{B}^{(r)}(A), \mathscr{B}^{(s)}(B)\right) .
$$

L'application $\operatorname{ad}(S)$ définie plus haut envoie $\operatorname{Hom}^{(i)}(\mathscr{B}(A), \mathscr{B}(B))$ dans $\operatorname{Hom}^{(i-1)}(\mathscr{B}(A), \mathscr{B}(B))$, et même surjectivement grâce au lemme 4.3 de [9]. Posons

$$
\begin{gathered}
\operatorname{Hom}_{S}^{(i)}(\mathscr{B}(A), \mathscr{B}(B)) \\
=\operatorname{Ker}\left(\operatorname{ad}(S): \operatorname{Hom}^{(i)}(\mathscr{B}(A), \mathscr{B}(B)) \rightarrow \operatorname{Hom}^{(i-1)}(\mathscr{B}(A), \mathscr{B}(B))[2]\right)
\end{gathered}
$$

et

$$
\operatorname{HC}_{(i)}^{n}(A, B)=H_{-n}\left(\operatorname{Hom}_{S}^{(i)}(\mathscr{B}(A), \mathscr{B}(B))\right) .
$$

Comme $\operatorname{Hom}(\mathscr{B}(A), \mathscr{B}(B))$ est le produit des sous-complexes $\operatorname{Hom}^{(i)}(\mathscr{B}(A), \mathscr{B}(B))$, 
alors $\operatorname{Hom}_{S}(\mathscr{B}(A), \mathscr{B}(B))$ se décompose:

$$
\operatorname{Hom}_{S}(\mathscr{B}(A), \mathscr{B}(B)) \cong \prod_{i \in \mathcal{Z}} \operatorname{Hom}_{S}^{(i)}(\mathscr{B}(A), \mathscr{B}(B)),
$$

ce qui donne la décomposition pour $\operatorname{HC}^{*}(A, B)$.

Regardons maintenant ce qui se passe lorsque l'on spécialise. La partie (1) du théorème 2.5 résulte du

LeMme 2.9. On $a$

a) $\operatorname{Hom}^{(i)}\left(C_{*}(A), C_{*}(k)\right) \cong \operatorname{Hom}\left(C_{*}^{(-i)}(A), k\right)$

b) $\operatorname{Hom}^{(i)}\left(C_{*}(k), C_{*}(B)\right) \cong C_{*}^{(i)}(B)$

c) $\operatorname{Hom}_{s}^{(i)}(\mathscr{B}(A), \mathscr{B}(k)) \cong \operatorname{Hom}\left(\mathscr{B}^{(-i)}(A), k\right)$

d) $\operatorname{Hom}_{S}^{(i)}(\mathscr{B}(k), \mathscr{B}(B)) \cong \mathscr{B}^{-(i)}(B)$.

DÉmonstration. Montrons d'abord (a) et (b). Pour l'anneau de base $k$, on sait que

$$
C_{*}(k)=C_{0}^{(0)}(k)=k
$$

ce qui donne

$$
\operatorname{Hom}^{(i)}\left(C_{*}(k), C_{*}(B)\right)=C_{*}^{(i)}(B) \text { et } \operatorname{Hom}^{(i)}\left(C_{*}(A), C_{*}(k)\right)=\operatorname{Hom}\left(C_{*}^{(-i)}(A), k\right) \text {. }
$$

Par conséquent, on a: $\mathrm{HH}_{(i)}^{*}(k, B)=\mathrm{HH}_{-*}^{(i)}(\mathrm{B})$. Quant à la décomposition de Gerstenhaber-Schack [5] de la cohomologie de Hochschild de $A$ à coefficients dans le bimodule $\operatorname{Hom}(A, k)$, elle s'écrit

$$
\mathrm{HH}^{*}(A)=\oplus_{i \leqq 0} \mathrm{HH}_{(i)}^{*}(A, k) .
$$

Passons aux points (c) et (d). D'après (2.10), un calcul rapide montre que $\mathscr{B}^{(i)}(k)$ est un complexe concentré en degré $2 i$

$$
\mathscr{B}^{(i)}(k)=k[2 i] .
$$

Lorsque $i \geqq 1, S: \mathscr{B}^{(i)}(k) \rightarrow \mathscr{B}^{(i-1)}(k)[2]$ est l'identité de $k[2 i]$.

Montrons (c)

Posons $B=k$. Alors

$$
\operatorname{Hom}^{(i)}(\mathscr{B}(A), \mathscr{B}(k))=\prod_{r \geqq-i} \operatorname{Hom}\left(\mathscr{B}^{(r)}(A), k\right)[2(r+i)] .
$$

Soit $f_{r} \in \operatorname{Hom}\left(\mathscr{B}^{(r)}(A), k\right)$. Alors

$$
\operatorname{ad}(S)\left(\ldots, f_{r}, f_{r-1}, \ldots\right)=\left(\ldots, f_{r}-f_{r-1} S, \ldots\right)=0
$$

si et seulement $f_{r}=f_{r-1} S$ pour tout $r$. Par conséquent: $f_{r}=f_{-i} S^{r+i}$. Il en résulte 
que si $f=\left(\ldots, f_{r}, \ldots\right)$ est dans $\operatorname{Hom}_{S}^{(i)}(\mathscr{B}(A), \mathscr{B}(k))$, alors $f$ est déterminé par $f_{-i}$ et donc, on a la partie (c) de lemme.

Pour finir, montrons (d).

Rappelons l'isomorphisme

$$
\phi: \mathscr{B}_{n}^{-}(A)=\prod_{p \geqq 0} C_{n+2 p}(B) \rightarrow \operatorname{Hom}_{S}(\mathscr{B}(k), \mathscr{B}(B))_{n}
$$

de [9] §1 donné par

$$
\phi\left(\left(x_{p}\right)_{p}\right)\left(u_{r}\right)=\sum_{k \geqq 0} u_{k} \otimes x_{r-k}
$$

pour $x_{p} \in C_{n+2 p}(B)$ et pour tout $\mathrm{p} \geqq 0$.

Comme $u_{r}$ est le générateur de $\mathscr{B}^{(r)}(k)$, alors $\left(\phi\left(x_{p}\right)_{p}\right)$ appartient à $\operatorname{Hom}^{(i)}(\mathscr{B}(k), \mathscr{B}(B))=\prod_{r} \operatorname{Hom}\left(\mathscr{B}^{(r)}(k), \mathscr{B}^{(r+i)}(B)\right)$ si et seulement si pour tout $r$ et tout $k$

$$
u_{k} \otimes x_{r-k} \in \mathscr{B}^{(r+i)}(B)
$$

ce qui est équivalent à $x_{r} \in C^{(r+i)}(B)$. Par conséquent, $\left(x_{p}\right)_{p} \in \mathscr{B}^{-(i)}(B)$ si et seulement si $\phi\left(\left(x_{p}\right)_{p}\right) \in \operatorname{Hom}_{S}^{(i)}(\mathscr{B}(k), \mathscr{B}(B))$.

Démontrons maintenant la longue suite exacte du théorème. Elle résulte du lemme suivant.

LEMME 2.11. Sous les hypothèses précédentes et si $A$ est projectif sur $k$, alors il existe pour tout entier $i$ une suite exacte de la forme $0 \rightarrow \operatorname{Hom}_{S}^{(i+1)}(\mathscr{B}(A), \mathscr{B}(B))[-2] \stackrel{s}{\rightarrow} \operatorname{Hom}_{S}^{(i)}(\mathscr{B}(A), \mathscr{B}(B)) \stackrel{I}{\rightarrow} \operatorname{Hom}^{(i)}(C(A), C(B)) \rightarrow 0$

DÉmONSTRATION. On procède comme pour la proposition I.2.1 de [12] et on obtient le diagramme de suites exactes

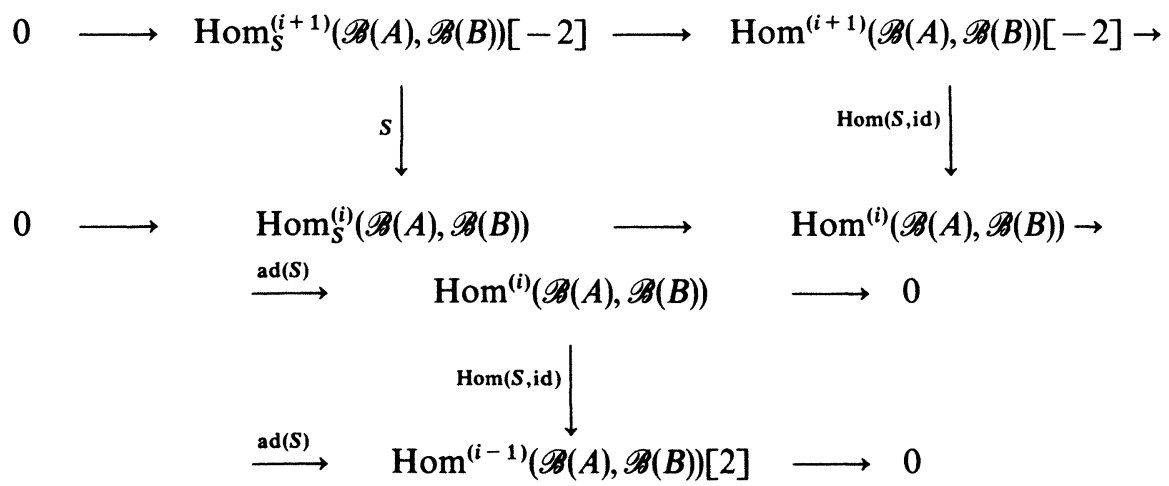

La flèche verticale du milieu est injective de conoyau 


$$
\operatorname{Hom}^{(i)}(C(A), \mathscr{B}(B))=\prod_{s-r=i} \operatorname{Hom}\left(C^{(r)}(A), \mathscr{B}^{(s)}(B)\right) .
$$

On termine en notant que $\operatorname{Hom}^{(i)}(C(A), C(B))$ est le noyau de

$$
\operatorname{Hom}^{(i)}(C(A), \mathscr{B}(B)) \stackrel{\text { Hom(id,S) }}{\longrightarrow} \mathrm{Hom}^{(i-1)}(C(A), \mathscr{B}(B))[2] .
$$

\section{Compatibilité de la décomposition et du produit.}

Dans ce paragraphe, nous démontrons le

THÉORÈME 3.1. Soit $k$ un anneau commutatif contenant $\mathrm{Q}$. Si $A_{1}, A_{2}, B_{1}, B_{2}$ et $C$ sont des $k$-algèbres commutatives, alors le produit en cohomologie cyclique bivariante est somme directe d'applications $k$-linéaires

$$
\mathrm{HC}_{(i)}^{*}\left(A_{1}, B_{1} \otimes C\right) \otimes \mathrm{HC}_{(j)}^{*}\left(C \otimes A_{2}, B_{2}\right) \rightarrow \mathrm{HC}_{(i+j)}^{*}\left(A_{1} \otimes A_{2}, B_{1} \otimes B_{2}\right) .
$$

DÉMONSTRATION. Eu égard à la définition du produit en cohomologie cyclique bivariante, il suffit de montrer

a) que le produit de composition

$$
\mathrm{HC}^{*}(A, B) \otimes \mathrm{HC}^{*}(B, C) \stackrel{\cup}{\rightarrow} \mathrm{HC}^{*}(A, C)
$$

est compatible avec la décomposition du paragraphe 1 ,

b) que les applications

$$
L(A): \mathrm{HC}^{*}(B, C) \rightarrow \mathrm{HC}^{*}(A \otimes B, A \otimes C)
$$

et

$$
R(A): \mathrm{HC}^{*}(B, C) \rightarrow \mathrm{HC}^{*}(B \otimes A, C \otimes A)
$$

(cf. proposition $5.3 \mathrm{de}$ [9]) sont aussi compatibles avec la décomposition.

La partie (a) ne présente pas de difficultés puisque clairement, si on compose un élément de $\operatorname{Hom}_{S}^{(i)}(\mathscr{B}(A), \mathscr{B}(B))$ avec un élément de $\operatorname{Hom}_{S}^{(j)}(\mathscr{B}(B), \mathscr{B}(C))$, on obtient un élément de $\operatorname{Hom}_{S}^{(i+j)}(\mathscr{B}(A), \mathscr{B}(C))$.

Démontrons maintenant que l'application $L(A)$ envoie $\mathrm{HC}_{(i)}^{*}(B, C)$ dans $\mathrm{HC}_{(i)}^{*}(A \otimes B, A \otimes C)$. Soit $f$ un élément de $\operatorname{Hom}_{S}^{(i)}(\mathscr{B}(B), \mathscr{B}(C))$ : c'est une famille $\left(f^{r}\right)_{r}$ d'applications

$$
f^{r}: \mathscr{B}^{(r)}(B) \rightarrow \mathscr{B}^{(s)}(C)
$$

pour $s=i+r$, telles que $S f^{r}=f^{r-1} S$. Soit $F^{r}$ l'application $F^{r}:(\mathscr{B}(A) \otimes \mathscr{B}(B))^{r}=\oplus_{u+v=r}\left(\mathscr{B}^{(u)}(A) \otimes \mathscr{B}^{(v)}(B)\right) \rightarrow \oplus_{u+v=r}\left(\mathscr{B}^{(u)}(A) \otimes \mathscr{B}^{(v+i)}(C)\right)$ définie par 


$$
F^{r}=\bigoplus_{u+v=r} \mathrm{id}^{u} \otimes f^{v}
$$

où id ${ }^{u}$ est l'identité de $\mathscr{B}^{(u)}(A)$ (on a $S \mathrm{id}^{u}=\mathrm{id}^{u-1} S$ ). Un calcul immédiat montre que $(S \otimes 1-1 \otimes S) F^{r}=F^{r-1}(S \otimes 1-1 \otimes S)$. Le théorème 1.7 de [10] nous assure que

$$
\mathscr{B}(C(A) \otimes C(B))=\operatorname{Ker}(S \otimes 1-1 \otimes S: \mathscr{B}(A) \otimes \mathscr{B}(B) \rightarrow \mathscr{B}(A) \otimes \mathscr{B}(C)[2]) .
$$

Posons $\mathscr{B}^{(r)}(C(A) \otimes C(B))=\operatorname{Ker}\left(S \otimes 1-1 \otimes S:(\mathscr{B}(A) \otimes \mathscr{B}(B))^{r} \rightarrow(\mathscr{B}(A) \otimes\right.$ $\left.\mathscr{B}(B))^{r-1}[2]\right)$. De façon analogue, on a $\mathscr{B}^{(s)}(C(A) \otimes C(C))$. On obtient un diagramme commutatif à lignes exactes

$$
\begin{aligned}
& 0 \longrightarrow \mathscr{B}^{(r)}(C(A) \otimes C(B)) \longrightarrow \bigoplus_{u+v=r}\left(\mathscr{B}^{(u)}(A) \otimes \mathscr{B}^{(v)}(B)\right) \rightarrow \\
& 0 \longrightarrow \mathscr{B}^{(s)}(C(A) \otimes C(C)) \longrightarrow \bigoplus_{u+v=r}\left(\mathscr{B}^{(u)}(A) \otimes \mathscr{B}^{(v+i)}(C)\right) \rightarrow \\
& \stackrel{s \otimes 1-1 \otimes s}{\longrightarrow} \bigoplus_{u+v=r-1}^{\longrightarrow}\left(\mathscr{B}^{(u)}(A) \otimes \mathscr{B}^{(v)}(B)\right)[2] \longrightarrow 0 \\
& \stackrel{s \otimes 1-1 \otimes s}{\longrightarrow} \underset{u+v=r-1}{\longrightarrow}\left(\mathscr{B}^{(u)}(A) \otimes \mathscr{B}^{(v+i)}(C)[2] \longrightarrow\right.
\end{aligned}
$$

Cela induit une application

$$
\tilde{F}^{r}: \mathscr{B}^{(r)}(C(A) \otimes C(B)) \rightarrow \mathscr{B}^{(s)}(C(A) \otimes C(C)) .
$$

Calculons $S F^{r}$. Sur le noyau $\mathscr{B}^{(r)}(C(A) \otimes C(B)), S$ est représenté au choix par $S \otimes 1$ ou $1 \otimes S$.

On a:

$$
\begin{aligned}
S \tilde{F}^{r} & =(1 \otimes S)\left(\oplus_{u+v=r} \mathrm{id}^{u} \otimes f^{v}\right) \\
& =\oplus_{u+v=r} \mathrm{id}^{u} \otimes S f^{r} \\
& =\oplus_{u+v=r} \mathrm{id}^{u} \otimes f^{r-1} S \\
& =\left(\oplus_{u+v=r-1} \mathrm{id}^{u} \otimes f^{v}\right)(1 \otimes S)=\tilde{F}^{r-1} S .
\end{aligned}
$$

Donc $\tilde{F}=\left(\widetilde{F}^{r}\right)_{r}$ définit un élément de 
$\operatorname{Ker}\left(\operatorname{ad} S: \operatorname{Hom}^{(i)}(\mathscr{B} C(A) \otimes C(B)), \mathscr{B}(C(A) \otimes C(C))\right) \rightarrow$

$$
\left.\mathrm{Hom}^{(i-1)}(\mathscr{B}(C(A) \otimes C(B)), \mathscr{B}(C(A) \otimes C(C)))[2]\right) .
$$

Nous faisons maintenant appel à [14] $\$ 7$ où il est montré que le "shuffleproduit" induit des $S$-équivalences entre $\mathscr{B}^{(r)}(C(A) \otimes C(B))$ et $\mathscr{B}^{(r)}(A \otimes B)$ ainsi qu'entre $\mathscr{B}^{(s)}(C(A) \otimes C(C))$ et $\mathscr{B}^{(s)}(A \otimes C)$. Il en résulte que $\widetilde{F}$ définit un élément de $\operatorname{Hom}_{S}^{(i)}(\mathscr{B}(A \otimes B), \mathscr{B}(A \otimes C))$.

La démonstration est analogue pour $R(A)$.

On termine la démonstration du théorème en rappelant que le produit

$$
\mathrm{HC}^{*}\left(A_{1}, B_{1} \otimes C\right) \otimes \mathrm{HC}^{*}\left(C \otimes A_{2}, B_{2}\right) \rightarrow \mathrm{HC}^{*}\left(A_{1} \otimes A_{2}, B_{1} \otimes B_{2}\right)
$$

est égal à $(\alpha, \beta) \rightarrow R\left(A_{2}\right)(\alpha) \cup L\left(B_{1}\right)(\beta)$.

\section{Le cas des algèbres lisses.}

Nous consacrons ce paragraphe à des exemples de calculs de groupes $\mathrm{HC}_{(i)}^{*}(A, B)$. Nous supposons ici que $k$ est un corps commutatif.

La plupart des algèbres dont on sait calculer l'homologie cyclique vérifient la propriété $(P)$ de [10]. Rappelons qu'une algèbre $B$ vérifie cette propriété si le $\mathrm{HC}_{*}(k)$-comodule $\mathrm{HC}_{*}(B)$ est somme d'un comodule étendu $\mathrm{HC}_{*}(k) \otimes U_{*}$ et d'un comodule trivial $V_{*}$ (ici $U_{*}$ et $V_{*}$ sont des $k$-modules gradués). On suppose $B$ commutative. De plus on suppose que $U_{*}$ et $V_{*}$ sont bigradués:

$$
U_{*}=\oplus_{p \geqq 0} U_{*}^{(p)} \text { et } V_{*}=\oplus_{p \geqq 0} V_{*}^{(p)}
$$

et que la décomposition de $\mathrm{HC}_{*}(B)$ est compatible à la bigraduation. Comme $\mathrm{HC}_{n}(k)=\mathrm{HC}_{n}^{\mathrm{in} / 2 \mathrm{I}}(k)$, on a:

$$
\mathrm{HC}_{n}^{(i)}(B)=\oplus_{p \geqq 0} U_{n-2 p}^{(i-p)} \oplus V_{n}^{(i)}
$$

Proposition 4.1. Sous les hypothèses précédentes, pour toute algèbre commutative A sur un corps commutatif $k$, on a l'isomorphisme

$$
\begin{aligned}
& H C_{(i)}^{n}(A, B) \\
& =\prod_{p, r \geqq 0} \operatorname{Hom}\left(\operatorname{HC}_{p}^{(r)}(A), U_{p-n}^{(i+r)}\right) \oplus \prod_{p, r \geqq 0} \operatorname{Hom}\left(\operatorname{HH}_{p}^{(r)}(A), V_{p-n-1}^{(i+r-1)}\right) .
\end{aligned}
$$

DÉmonstration. Par définition (voir 2.8), on a la suite exacte $0 \rightarrow \operatorname{Hom}_{S}^{(i)}(\mathscr{B}(A), \mathscr{B}(B)) \rightarrow \operatorname{Hom}^{(i)}(\mathscr{B}(A), \mathscr{B}(B)) \stackrel{\text { ad(S) }}{\longrightarrow} \operatorname{Hom}^{(i-1)}(\mathscr{B}(A), \mathscr{B}(B))[2] \rightarrow 0$ d'où la longue suite exacte 
$\ldots \rightarrow H_{-n+1}\left(\prod_{r} \operatorname{Hom}\left(\mathscr{B}^{(r)}(A), \mathscr{B}^{(r+i)}(B)\right)\right) \stackrel{(\operatorname{ad}(S))_{-n+1}^{(i)}}{\longrightarrow}$
$\rightarrow H_{-n+1}\left(\prod_{r} \operatorname{Hom}\left(\mathscr{B}^{(r)}(A), \mathscr{B}^{(r+i-1)}(B)\right)[2]\right) \rightarrow \mathrm{HC}_{(i)}^{n}(A, B) \rightarrow$ $\rightarrow H_{-n}\left(\prod_{r} \operatorname{Hom}\left(\mathscr{B}^{(r)}(A), \mathscr{B}^{(r+i)}(B)\right)\right) \stackrel{(\operatorname{ad}(S))_{-n}^{(i)}}{\longrightarrow}$

$$
\rightarrow H_{-n}\left(\prod_{r} \operatorname{Hom}\left(\mathscr{B}^{(r)}(A), \mathscr{B}^{(r+i-1)}(B)\right)[2]\right) \rightarrow \ldots
$$

Soit $X_{(i)}^{n}=\operatorname{Ker}\left((\operatorname{ad}(S))_{-n}^{(i)}\right)$ et $Y_{(i)}^{n}=\operatorname{Coker}\left(\left(\operatorname{ad}(S)_{-n+1}^{(i)}\right)\right.$ et donc, on a un isomorphisme d'espaces vectoriels

$$
\mathrm{HC}_{(i)}^{n}(A, B)=X_{(i)}^{n} \oplus Y_{(i)}^{n} .
$$

Or d'après la formule des coefficients universels sur un corps, on a

$$
H_{-n}\left(\prod_{r} \operatorname{Hom}\left(\mathscr{B}^{(r)}(A), \mathscr{B}^{(r+i)}(B)\right)\right)=\prod_{r} \operatorname{Hom}\left(\operatorname{HC}^{(r)}(A), \mathrm{HC}^{(r+i)}(B)\right)_{-n} .
$$

Par hypothèse

$$
\mathrm{HC}_{n}^{(i)}(B)=\oplus_{p \geqq 0} U_{n-2 p}^{(i-p)} \oplus V_{n}^{(i)} .
$$

Calculons d'abord le noyau et le conoyau de

$$
\prod_{r} \operatorname{Hom}\left(\mathrm{HC}^{(r)}(A), V^{(r+i)}\right) \stackrel{\text { adS }}{\longrightarrow} \prod_{r} \operatorname{Hom}\left(\mathrm{HC}^{(r)}(A), V^{(r+i-1)}\right)[2]
$$

Puisque $S$ est nul sur $V^{(i)}$, l'application précédente est le produit des applications

$$
\prod_{r} \operatorname{Hom}\left(\mathrm{HC}^{(r)}(A) \stackrel{s}{\rightarrow} \mathrm{HC}^{(r-1)}(A), V^{(r+i)}\right)
$$

dont le noyau est $\prod_{r} \operatorname{Hom}\left(\operatorname{Coker}\left(\mathrm{HC}^{(r)}(A) \stackrel{s}{\rightarrow} \mathrm{HC}^{(r-1)}(A)\right), V^{(r+i)}\right)$ et le conoyau est $\prod_{r} \operatorname{Hom}\left(\operatorname{Ker}\left(\mathrm{HC}^{(r)}(A) \stackrel{s}{\rightarrow} \mathrm{HC}^{(r-1)}(A)\right), V^{(r+i)}\right)$.

Pour calculer le noyau et le conoyau de

$$
\prod_{r} \operatorname{Hom}\left(\mathrm{HC}^{(r)}(A), \bigoplus_{p \geqq 0} U^{(r+i-p)}[2 p]\right) \rightarrow \prod_{r} \operatorname{Hom}\left(\mathrm{HC}^{(r-1)}(A), U^{(r+i-p)}[2 p]\right)[2]
$$

on remarque que cette application est en facteur direct dans

$$
\operatorname{Hom}(\mathrm{HC}(A), k[u] \otimes U) \stackrel{\operatorname{ad}(S)}{\longrightarrow} \operatorname{Hom}(H C(A), k[u] \otimes U)[2]
$$

dont on sait que c'est surjectif, car $k[u] \otimes U$ est $S$-module étendu (voir [9]), et 
dont le noyau est $\operatorname{Hom}(\operatorname{HC}(A), U)$ via l'isomorphisme

$$
\phi: \operatorname{Hom}(\mathrm{HC}(A), U) \rightarrow \operatorname{Hom}_{S}(\mathrm{HC}(A), k[u] \otimes U)
$$

de [9] §1. Il suffit donc de trouver l'image inverse par $\phi$ de

$$
\mathrm{Hom}^{(i)}(\mathrm{HC}(A), k[u] \otimes U) \text {. }
$$

Un calcul montre que c'est $\prod_{r} \operatorname{Hom}\left(\operatorname{HC}^{(r)}(A), U^{(r+i)}\right)$.

Pour résumer

$$
\begin{aligned}
& X_{(i)}^{n}=\prod_{r, k \geqq 0} \operatorname{Hom}\left(\mathrm{HC}_{k}^{(r)}(A), U_{k-n}^{(r+i)}\right) \oplus \\
& \oplus \prod_{r, k \geqq 0} \operatorname{Hom}\left(\operatorname{Ker}\left(\mathrm{HC}_{k}^{(r)}(A) \stackrel{s}{\rightarrow} \operatorname{HC}_{k-2}^{(r-1)}(A)\right), V_{k-n-1}^{(r+i-1)}\right)
\end{aligned}
$$

et

$$
Y_{(i)}^{n}=\prod_{r, k \geqq 0} \operatorname{Hom}\left(\operatorname{Coker}\left(\mathrm{HC}_{k+2}^{(r+1)}(A) \stackrel{s}{\rightarrow} \mathrm{HC}_{k}^{(r)}(A)\right), V_{k-n-1}^{(r+i-1)}\right) .
$$

Pour finir, on utilise les suites exactes dérivées de la longue suite exacte de Connes

$0 \rightarrow \operatorname{Coker}\left(\mathrm{HC}_{k+2}^{(r+1)}(A) \stackrel{s}{\rightarrow} \mathrm{HC}_{k}^{(r)}(A)\right) \rightarrow \mathrm{HH}_{k}^{(r)}(A) \rightarrow$

$$
\rightarrow \mathrm{Ker}\left(\mathrm{HC}_{k}^{(r)}(A) \rightarrow \mathrm{HC}_{k-2}^{(r-1)}(A)\right) \rightarrow 0 .
$$

En mettant tout ensemble, on trouve les formules de la proposition.

En faisant $B=k$, on retrouve

$$
\mathrm{HC}_{(i)}^{n}(A, k)=\operatorname{Hom}\left(\mathrm{HC}_{n}^{(-i)}(A), k\right)
$$

ce qu'on savait d'après le théorème 2.5. En posant $A=k$, on retrouve:

$$
\mathrm{HC}_{n}^{-(i)}(B)=\prod_{p \geqq 0} U_{n+2 p}^{(i+p)} \oplus V_{n-1}^{(k)} .
$$

4.2 Application aux algèbres lisses. Une algèbre lisse $B$ en caractéristique nulle a la propriété $(\mathrm{P})$ avec

$$
U_{n}=U_{n}^{(n)}=H_{D R}^{n}(B) \text { et } V_{n}=V_{n}^{(n)}=d \Omega_{B}^{n} .
$$

En appliquant la formule précédente, on obtient:

$$
\begin{aligned}
& \operatorname{HC}_{(i)}^{n}(A, B) \cong \prod_{p \geqq 0} \operatorname{Hom}\left(\operatorname{HC}_{p}^{(p-n-i)}(A), H_{D R}^{p-n}(B)\right) \oplus \\
& \oplus \prod_{p \geqq 0} \operatorname{Hom}\left(\operatorname{HH}_{p}^{(p-n-i)}(A), d \Omega_{B}^{p-n-1}\right) .
\end{aligned}
$$


Supposons maintenant $A$ et $B$ lisses sur un corps de caractéristique zéro. On sait alors que

$$
\mathrm{HC}_{n}^{(i)}(A)= \begin{cases}H_{D R}^{n}(A) \oplus d \Omega_{A}^{n} & \text { si } i=n \\ H_{D R}^{2 i-n}(A) & \text { si } i<n\end{cases}
$$

et que

$$
\mathrm{HH}_{n}^{(i)}(A)= \begin{cases}\Omega_{A}^{n} & \text { si } i=n \\ 0 & \text { sinon }\end{cases}
$$

La formule (4.3) devient:

$$
\mathrm{HC}_{(i)}^{n}(A, B)= \begin{cases}\prod_{r} \operatorname{Hom}\left(H_{D R}^{r}(A), H_{D R}^{r+n+2 i}(B)\right) & \text { si } i>-n \\ 0 & \text { si } i<-n \\ \prod_{r} \operatorname{Hom}\left(H_{D R}^{r}(A), H_{D R}^{r-n}(B)\right) \oplus & \\ \oplus \prod_{r} \operatorname{Hom}\left(d \Omega_{A}^{r}, H_{D R}^{r-n}(B)\right) \oplus & \\ \oplus \prod_{r} \operatorname{Hom}\left(\Omega_{A}^{r}, d \Omega_{B}^{r-n-1}\right) & \text { si } i=-n\end{cases}
$$

\section{Cohomologie cyclique bivariante périodique.}

Dans ce paragraphe nous donnons une décomposition de l'homologie cyclique périodique, puis de la cohomologie cyclique bivariante périodique dans le cas des algèbres lisses.

Rappelons ([6],[10]) que l'homologie cyclique périodique $\mathrm{HP}_{*}(A)$ d'une $k$-algèbre $A$ est l'homologie du complexe Z-gradué

$$
\ldots \stackrel{b+B}{\longrightarrow} \prod_{p \geqq 0} C_{2 p}(A) \stackrel{b+B}{\longrightarrow} \prod_{p \geqq 0} C_{2 p+1}(A) \stackrel{b+B}{\longrightarrow} \prod_{p \geqq 0} C_{2 p}(A) \stackrel{b+B}{\longrightarrow} \ldots
$$

Ce complexe est isomorphe à $\lim (A)[2 m]$, le système projectif étant donné par la surjection naturelle $S$ de $\mathscr{B}(A)$ sur $\mathscr{B}(A)[2]$.

Supposons $A$ commutative, alors $\mathscr{B}(A)=\oplus \mathscr{B}^{(i)}(A)$ (cf. 1.4). En posant $\mathscr{B}_{n}^{(i)}(A)=0$ si $i<0$ ou $n_{S}<0$ ou $i>n$, on a, pour tout couple d'entiers $(i, n)$ un système projectif $\left\{\ldots \stackrel{s}{\rightarrow} \mathscr{B}_{n}^{(i)}(A) \stackrel{s}{\rightarrow} \mathscr{B}_{n-2}^{(i-1)}(A) \stackrel{s}{\rightarrow} \ldots\right\}$. L'homologie du complexe limite projective est notée $\operatorname{HP}_{*}^{(i)}(A)$. Par définition même on a

$$
\operatorname{HP}_{2 n}^{(i)}(A) \cong \operatorname{HP}_{0}^{(i-n)}(A) \text { et } \operatorname{HP}_{2 n+1}^{(i)}(A) \cong \operatorname{HP}_{1}^{(i-n)}(A)
$$

Proposition 5.2. Le module $\mathrm{HP}_{*}(A)$ est $(\mathrm{Z} / 2) \times \mathrm{Z}$-gradué et on a:

$$
\mathrm{HP}_{*}(A) \cong \prod_{i \in Z} \operatorname{HP}_{*}^{(i)}(A) \text {. }
$$


DÉmonstration. Comme il n'y a qu'un nombre fini de $\mathscr{B}_{n}^{(i)}(A)$ non nuls, $\mathscr{B}_{n}(A)$ est isomorphe au produit pour tous les entiers $i$ des $\mathscr{B}_{n}^{(i)}(A)$. On utilise ensuite le fait que le produit commute aux limites projectives et à l'homologie.

5.3. EXEMPLE. Si $A$ est une algèbre lisse sur un corps de caractéristique zéro, on déduit du paragraphe 4.2 que

$$
\mathrm{HP}_{n}^{(i)}(A) \cong H_{D R}^{2 i-n}(A) \quad \text { si } i<n
$$

et donc

$$
\begin{array}{ll}
\mathrm{HP}_{0}^{(i)}(A) \cong H_{D R}^{2 i}(A) & \text { si } i \geqq 0 \\
\operatorname{HP}_{0}^{(i)}(A) \cong 0 & \text { si } i<0 \\
\mathrm{HP}_{1}^{(i)}(A) \cong H_{D R}^{2 i-1}(A) & \text { si } i \geqq 1 \\
\mathrm{HP}_{1}^{(i)}(A) \cong 0 & \text { si } i<1 .
\end{array}
$$

5.4. Cas bivariant. Dans [9], Jones et Kassel définissent la cohomologie cyclique bivariante périodique $\operatorname{HP}^{*}(A, B)$ de deux algèbres $A$ et $B$ comme étant la limite inductive du système

$$
\left\{\ldots \stackrel{s}{\rightarrow} \mathrm{HC}^{*}(A, B) \stackrel{s}{\rightarrow} \mathrm{HC}^{*+2}(A, B) \stackrel{s}{\rightarrow} \ldots\right\}
$$

Si $A$ et $B$ sont commutatives, il est naturel de poser:

$$
\mathrm{HP}_{(i)}^{n}(A, B)=\underline{\lim }\left\{\ldots \stackrel{s}{\longrightarrow} \mathrm{HC}_{(i)}^{n}(A, B) \stackrel{s}{\rightarrow} \mathrm{HC}_{(i-1)}^{n+2}(A, B) \stackrel{\mathrm{s}}{\longrightarrow} \ldots\right\}
$$

On a les isomorphismes

$$
\operatorname{HP}_{(i)}^{2 n}(A, B) \cong \operatorname{HP}_{(i+n)}^{0}(A, B) \text { et } \operatorname{HP}_{(i)}^{2 n+1}(A, B) \cong \operatorname{HP}_{(i+n)}^{1}(A, B)
$$

Cependant, comme la limite inductive ne commute pas aux produits, la cohomologie cyclique bivariante périodique n'est pas en général le produit des $\mathrm{HP}_{(i)}^{*}(A, B)$.

Si on spécialise, on a

$$
\operatorname{HP}_{(i)}^{n}(A, k) \cong \operatorname{HP}_{(-i)}^{n}(A):=\operatorname{Hom}\left(\operatorname{HP}_{n}^{(i)}(A), k\right) \text { et } \operatorname{HP}_{(i)}^{n}(k, B) \cong \operatorname{HP}_{-n}^{(i)}(B)
$$

5.5 EXEMPLE. Si $A$ et $B$ sont des algèbres lisses sur un corps de caractéristique zéro, on déduit de 4.4 que

$$
\operatorname{HP}_{(i)}^{n}(A, B) \cong \prod_{r} \operatorname{Hom}\left(H_{D R}^{r}(A), H_{D R}^{r+n+2 i}(B)\right)
$$

Dans ce cas et d'après le calcul de [9] exemple 8.3, on voit que $\operatorname{HP}^{n}(A, B)$ est le produits des $\operatorname{HP}_{(i)}^{n}(A, B)$.

\section{Renormalisation de la cohomologie cyclique bivariante.}

On commence par rappeler certaines notations de [9] et [10]. Soit $k$ un anneau commutatif. Un complexe mixte $(M, b, B)$ est un complexe de $k$-modules $(M, b)$ 
muni d'un endomorphisme $B: M \rightarrow M$ de degré +1 vérifiant:

$$
b B+B b=B^{2}=0 .
$$

Au complexe mixte $(M, b, B)$ on associe le complexe $\mathscr{B}(M)=k[u] \otimes M$, où $u$ est une indéterminée en degré 2 , muni de la différentielle

$$
d\left(u^{n} \otimes m\right)= \begin{cases}u^{n} \otimes b m+u^{n-1} \otimes B m & \text { si } n \geqq 1 \\ u^{n} \otimes b m & \text { si } n=0 .\end{cases}
$$

La projection naturelle $S: \mathscr{B}(M) \rightarrow \mathscr{B}(M)[2]$ donnée par

$$
S\left(u^{n} \otimes m\right)= \begin{cases}u^{n-1} \otimes m & \text { si } n \geqq 1 \\ 0 & \text { si } n=0\end{cases}
$$

est un morphisme de complexes qui fait de $\mathscr{B}(M)$ un $S$-module, i.e. un complexe borné inférieurement muni d'un endomorphisme de degré -2 .

Soit $l$ un élément inversible de $k$. Au complexe mixte $M=(M, b, B)$, on associe le complexe mixte

$$
M_{l}=(M, b, l B) .
$$

Si $P$ est un $S$-module, on note $P(l)$ le $S$-module qui a même complexe sous-jacent, mais dont l'operateur $S$ est égal à $l S$. Le résultat qui suit est un cas particulier de [13], Lemme A.2.

Lemme 6.1. Soit $(M, b, B)$ un complexe mixte et l un élément inversible de $k$. Alors l'application

$$
\sigma_{l}: \mathscr{B}\left(M_{l}\right) \rightarrow \mathscr{B}(M)(1 / l)
$$

définie par $\sigma_{l}\left(u^{n} \otimes m\right)=l^{n} u^{n} \otimes m$ est un isomorphisme de S-modules.

DÉmonstration. On a:

$$
d \sigma_{l}\left(u^{n} \otimes m\right)=d\left(l^{n} u^{n} \otimes m\right)=l^{n} u^{n} \otimes b m+l^{n} u^{n-1} \otimes B m .
$$

Par ailleurs

$$
\begin{aligned}
\sigma_{l} d\left(u^{n} \otimes m\right) & =\sigma_{l}\left(u^{n} \otimes b m+u^{n-1} \otimes l B m\right) \\
& =l^{n} u^{n} \otimes b m+l^{n-1} u^{n-1} \otimes l B m=d \sigma_{l}\left(u^{n} \otimes m\right) .
\end{aligned}
$$

Donc $\sigma_{l}$ est un isomorphisme de complexes d'inverse $\sigma_{1 / l}$. On vérifie ensuite que

$$
\frac{1}{l} S \sigma_{l}=\sigma_{l} S .
$$

Il en résulte que l'homologie de $\mathscr{B}\left(M_{l}\right)$ ne dépend pas de $l$. 
Rappelons qu'on définit la cohomologie cyclique bivariante $\operatorname{HC}^{*}(M, N)$ de deux complexes mixtes $M$ et $N$ comme la cohomologie du complexe $\operatorname{Hom}_{S}(\mathscr{B}(M), \mathscr{B}(N))$ des applications graduées $f$ de $\mathscr{B}(M)$ dans $\mathscr{B}(N)$ vérifiant:

$$
f S=S f .
$$

Du lemme précédent, on tire aisément le

LEMME 6.2. Sous les hypothèses précédentes, soit $l, m$ deux éléments inversibles de $k$. On a alors les isomorphismes de complexes

$\operatorname{Hom}_{S}\left(\mathscr{B}\left(M_{l}\right), \mathscr{B}\left(N_{m}\right)\right) \cong \operatorname{Hom}_{S}\left(\mathscr{B}(M), \mathscr{B}\left(N_{m / l}\right)\right) \cong \operatorname{Hom}_{S}\left(\mathscr{B}\left(M_{l / m}\right), \mathscr{B}(N)\right)$.

On voit que les groupes précédents ne dépendent que du rapport $m / l$. On pose alors

$$
\begin{aligned}
\operatorname{HC}^{*}(M, N)(l) & =H_{-*}\left(\operatorname{Hom}_{S}\left(\mathscr{B}(M), \mathscr{B}\left(N_{l}\right)\right)=\right. \\
& =H_{-*}\left(\operatorname{Hom}_{S}(\mathscr{B}(M)(l), \mathscr{B}(N))\right) .
\end{aligned}
$$

On a évidemment:

$$
\mathrm{HC}^{*}(M, N)(1)=\mathrm{HC}^{*}(M, N) .
$$

Du lemme 6.2, on tire aussi la

Proposition 6.4. Sous les hypothèses précédentes, il existe un produit de composition gradué pour les complexes mixtes $L, M, N$

$$
\mathrm{HC}^{*}(L, M)(l) \otimes \mathrm{HC}^{*}(M, N)(m) \stackrel{u}{\rightarrow} \mathrm{HC}^{*}(L, N)(l m) .
$$

DÉmONSTRATION. Le produit est obtenu par composition de $\operatorname{Hom}_{S}(\mathscr{B}(L)$, $\left.\mathscr{B}\left(M_{l}\right)\right)$ et de $\operatorname{Hom}_{s}\left(\mathscr{B}(M), \mathscr{B}\left(N_{m}\right)\right) \cong \operatorname{Hom}_{S}\left(\mathscr{B}\left(M_{l}\right), \mathscr{B}\left(N_{l m}\right)\right)$.

Le reste du paragraphe est consacré à la question de savoir comment $\mathrm{HC}^{*}(M, N)(l)$ dépend de $l$.

Considérons $k$ comme le complexe mixte trivial $(k, 0,0)$. On a alors, en reprenant les notations de [9], la proposition:

Proposition 6.5. Pour tout complexe mixte $M$, tout élément inversible de $k$ et tout entier $n$, on a:

$$
\mathrm{HC}^{n}(M, k)(l) \cong \mathrm{HC}^{n}(M) \text { et } \mathrm{HC}^{n}(k, M)(l) \cong \mathrm{HC}_{-n}^{-}(M) .
$$

DÉMONSTRATION.

$$
\begin{aligned}
\operatorname{HC}^{n}(M, k)(l) & =H_{-n}\left(\operatorname{Hom}_{S}(\mathscr{B}(M)(l), \mathscr{B}(k))\right. \\
& =H_{-n}(\operatorname{Hom}(\mathscr{B}(M)(l), k)) \\
& \cong H_{-n}(\operatorname{Hom}(\mathscr{B}(M), k)) \quad \text { d'après le lemme 6.2. } \\
& \cong \mathrm{HC}^{n}(M)
\end{aligned}
$$


On démontre de même l'autre isomorphisme.

Voici une autre situation dans laquelle $\operatorname{HC}^{*}(M, N)(l)$ ne dépend pas de $l$. Supposons que $M$ soit un complexe mixte de la forme $M=(M, 0, B)$. Alors clairement l'application $x \mapsto x / l$ définit un isomorphisme de complexes mixtes de $M$ sur $M_{l}$, donc un isomorphisme de $S$-modules de $\mathscr{B}(M) \operatorname{sur} \mathscr{B}\left(M_{l}\right) \cong \mathscr{B}(M)(1 / l)$. On tire la

Proposition 6.6. Supposons qu'un complexe mixte $M=(M, b, B)$ soit quasiisomorphe à un complexe mixte $M^{\prime}$ de la forme $\left(M^{\prime}, 0, B^{\prime}\right)$. Alors, pour tout complexe mixte $N$ et tout linversible dans $k$, on a:

$$
\mathrm{HC}^{*}(M, N)(l) \cong \mathrm{HC}^{*}(M, N)
$$

si $M$ et $M^{\prime}$ sont projectifs sur $k$ et

$$
\operatorname{HC}^{*}(N, M)(l) \cong \mathrm{HC}^{*}(N, M)
$$

si $N$ est projectif sur $k$.

Démonstration. Puisque $\mathscr{B}\left(M_{l}^{\prime}\right)$ est isomorphe comme $S$-module à $\mathscr{B}\left(M^{\prime}\right)$, on voit que $\mathrm{HC}^{*}\left(M^{\prime}, N\right)(l)$ et $\mathrm{HC}^{*}\left(N, M^{\prime}\right)(l)$ ne dépendent pas de $l$. On termine en appliquant le corollaire 4.6 de [9].

EXEMPLE 6.7. La proposition précédente s'applique au cas où $M$ est le complexe mixte $(C(A), b, B)$ attaché à une algèbre commutative lisse $A$ sur un corps $k$ de caractéristique nulle. En effet, d'après [16], on peut prendre pour $M^{\prime}$ le complexe mixte $\left(\Omega_{A / k}^{*}, 0, d\right)$.

Nous terminons ce paragraphe avec un couple exact et une formule de coefficients universels pour $\mathrm{HC}^{*}(M, N)(l)$.

THÉORÈME 6.8. Soit $M$ et $N$ deux complexes mixtes et $l$ inversible dans $k$. Supposons $M$ projectif sur $k$, alors il existe une longue suite exacte

$$
\begin{aligned}
\ldots \stackrel{B}{\longrightarrow} \mathrm{HC}^{n-2}(M, N)(l) \stackrel{\mathrm{s}}{\rightarrow} \mathrm{HC}^{n}(M, N)(l) \stackrel{I}{\longrightarrow} \\
\stackrel{I}{\longrightarrow} \mathrm{HH}^{n}(M, N) \stackrel{B}{\longrightarrow} \mathrm{HC}^{n-1}(M, N)(l) \stackrel{s}{\rightarrow} \ldots
\end{aligned}
$$

DÉmONSTRATION. On applique la proposition I.2.1 de [12] aux complexes mixtes $M$ et $N_{l}$.

Rappelons avec [9] que si $P$ et $Q$ sont des $S$-modules, alors on pose

$$
\underline{\operatorname{Ext}}_{s}(P, Q)=\operatorname{Coker}(\operatorname{ad}(S): \operatorname{Hom}(P, Q)[-2] \rightarrow \operatorname{Hom}(P, Q))
$$

et

$$
\operatorname{Ext}_{s}^{*}(P, Q)=H_{-*}\left(\operatorname{Ext}_{s}(P, Q)\right)
$$


On a alors le théorème des coefficients universels:

THÉORÈME 6.9. Soit $M$ et $N$ deux complexes mixtes tels que $M$ et $\mathrm{HC}_{*}(M)$ soient projectifs sur $k$. Alors pour tout élément inversible l de $k$ et tout entier $n$, on a une suite exacte naturelle de la forme

$0 \rightarrow \operatorname{Ext}_{S}^{n+1}\left(\mathrm{HC}_{*}(M), \mathrm{HC}_{*}(N)\right) \rightarrow \mathrm{HC}^{n}(M, N)(l) \rightarrow \operatorname{Hom}_{S}\left(\mathrm{HC}_{*}(M), \mathrm{HC}_{*}(N)\right)_{-n} \rightarrow 0$.

DÉmONSTRATION. On applique le théorème 4.2 de [9] aux complexes mixtes $M$ et $N_{l}$. On obtient alors la suite exacte

$0 \rightarrow \operatorname{Ext}_{S}^{n+1}\left(\mathrm{HC}_{*}(M), \mathrm{HC}_{*}\left(N_{l}\right)\right) \rightarrow \mathrm{HC}^{n}(M, N)(l) \rightarrow \operatorname{Hom}_{S}\left(\mathrm{HC}_{*}(M), \mathrm{HC}_{*}\left(N_{l}\right)\right)_{-n} \rightarrow 0$.

Nous avons vu plus haut que

$$
\mathrm{HC}_{*}\left(N_{l}\right) \cong H_{*}\left(\mathscr{B}\left(N_{l}\right)\right) \cong H_{*}(\mathscr{B}(N)(1 / l))
$$

est isomorphe à $H_{*}(\mathscr{B}(N))(1 / l)=\mathrm{HC}_{*}(N)(1 / l)$ comme $S$-module.

Or l'application

$$
x \mapsto l^{n} x
$$

pour $x \in \mathrm{HC}_{2 n}(N)$ ou $x \in \mathrm{HC}_{2 n+1}(N)$, induit un isomorphisme

$$
\mathrm{HC}_{*}(N) \rightarrow \mathrm{HC}_{*}(N)(1 / l)
$$

de $S$-modules, ce qui permet de conclure.

Corollaire 6.10. Soit $k$ un corps commutatif et $M, N$ deux complexes mixtes. Alors pour tout élément inversible l de $k$,

$$
\mathrm{HC}^{*}(M, N)(l) \cong \mathrm{HC}^{*}(M, N)
$$

(l'isomorphisme est non canonique).

\section{Opérations d'Adams en cohomologie cyclique bivariante.}

Dans ce paragraphe, nous utilisons les constructions du paragraphe précédent pour étendre les opérations d'Adams de l'homologie cyclique à la cohomologie cyclique bivariante.

Rappelons que Loday [15] a construit sur le complexe de Hochschild $(C(A), b)$ d'une algèbre commutative $A$ des endomorphismes $\psi_{A}^{l}\left(l \in N^{*}\right)$ vérifiant:

$$
\begin{aligned}
& \text { i) } \psi_{A}^{l} b=b \psi_{A}^{l} \\
& \text { ii) } \psi_{A}^{l} B=l B \psi_{A}^{l} \\
& \text { iii) } \psi_{A}^{1}=\text { identité } \\
& \text { iv) } \psi_{A}^{l} \circ \psi_{A}^{m}=\psi_{A}^{m} \circ \psi_{A}^{l}=\psi_{A}^{l m} \text {. }
\end{aligned}
$$

Il en résulte que $\psi_{A}^{l}$ est un morphisme du complexe mixte $C(A)=(C(A), b, B)$ 
dans le complexe mixte $C(A)_{l}=(C(A), b, l B)$. D'où un 0-cycle de

$$
\operatorname{Hom}_{S}\left(\mathscr{B}(C(A)), \mathscr{B}\left(C(A)_{l}\right)\right.
$$

dont la cohomologie est $\mathrm{HC}^{*}\left(C(A), C(A)_{l}\right)$. Pour simplifier, étant données deux algèbres $A$ et $B$, nous poserons désormais

$$
\mathrm{HC}^{*}(A, B)(l)=\mathrm{HC}^{*}\left(C(A), C(B)_{l}\right) .
$$

De ce qui précède, on obtient:

Proposition 7.3. Les éléments $\left[\psi_{A}^{l}\right] \in \operatorname{HC}^{0}(A, A)(l)$ vérifient les propriétés suivantes:

i) $\left[\psi_{A}^{1}\right]=[\mathrm{id}] \in \mathrm{HC}^{0}(A, A)(1)=\mathrm{HC}^{0}(A, A)$

ii) dans $\mathrm{HC}^{0}(A, A)(l m)$, on a:

$$
\left[\psi_{A}^{l}\right] \cup\left[\psi_{A}^{m}\right]=\left[\psi_{A}^{m}\right] \cup\left[\psi_{A}^{l}\right]=\left[\psi_{A}^{l m}\right] .
$$

Avant d'aller plus loin, montrons que ceci permet de donner aux groupes $\mathrm{HH}^{*}(A, B)$ et $\mathrm{HH}^{*}(B, A)$ une structure de $\gamma$-anneau au sens d'Atiyah-Tall [1].

En effet, l'image de $\left[\psi_{A}^{l}\right]$ par l'application naturelle (cf. §6)

$$
I: \mathrm{HC}^{0}(A, A)(l) \rightarrow \mathrm{HH}^{0}(A, A)
$$

donne un élément $I\left[\psi_{A}^{l}\right] \in \mathrm{HH}^{0}(A, A)$ vérifiant les propriétés précédentes. Soit maintenant $B$ une algèbre quelconque. On note $L I \psi_{A}^{l}$ et $R I \psi_{A}^{l}$ les endomorphismes des $k$-modules gradués respectifs $\mathrm{HH}^{*}(A, B)$ et $\mathrm{HH}^{*}(B, A)$, définis par

$$
L I \psi_{A}^{l}(\alpha)=I\left[\psi_{A}^{l}\right] \cup \alpha \text { et } R I \psi_{A}^{l}(\beta)=\beta \cup I\left[\psi_{A}^{l}\right] .
$$

Utilisant ce qui précède et l'appendice de [15], on obtient aussitôt la

Proposition 7.4. Soit $A$ une algèbre commutative et $B$ une algèbre quelconque. Munissons $\mathrm{HH}^{*}(A, B)$ et $\mathrm{HH}^{*}((B, A)$ de la multiplication nulle. Alors les endomorphismes $\left(L I \psi_{A}^{l}\right)_{l}\left(\right.$ resp. $\left.\left(R I \psi_{A}^{l}\right)_{l}\right)$ munissent $\mathrm{HH}^{*}(A, B)\left(\right.$ resp. $\left.\mathrm{HH}^{*}(B, A)\right) d$ 'une structure de $\gamma$-anneau.

Pour obtenir un résultat du même type pour la cohomologie cyclique bivariante, il convenient d'introduire les groupes

$$
\widehat{H C}^{*}(A, B)=\bigoplus_{l \in \mathbb{N}} \mathrm{HC}^{*}(A, B)(l)
$$

Nous supposons dorénavant que $k$ est un corps de caractéristique nulle. Le corollaire 6.10 implique que $\widehat{H C}^{*}(A, B)$ est une somme directe d'une infinité de copies de $\mathrm{HC}^{*}(A, B)$.

Notons $L \psi_{A}^{l}\left(\right.$ resp. $\left.R \psi_{A}^{l}\right)$ les endomorphismes de $\widehat{H C} \widehat{C}^{*}(A, B)\left(\right.$ resp. de $\left.\widehat{H C} \widehat{C}^{*}(B, A)\right)$ donnés par 


$$
L \psi_{A}^{l}(\alpha)=\left[\psi_{A}^{l}\right] \cup \alpha \quad\left(\text { resp. } R \psi_{A}^{l}(\beta)=\beta \cup\left[\psi_{A}^{l}\right]\right) .
$$

On vérifie aussi que $L \psi_{A}^{1}$ et $R \psi_{A}^{1}$ sont l'identité et que

$$
L \psi_{A}^{l} \circ L \psi_{A}^{m}=L \psi_{A}^{m} \circ L \psi_{A}^{l}=L \psi_{A}^{l m}
$$

(mêmes relations pour $R \psi_{A}^{*}$ ). On résume avec:

THÉORÈME 7.7. Soit $A$ une algèbre commutative et $B$ une algèbre quelconque sur un corps commutatif $k$ de caractéristique nulle. Munissons $\widehat{\mathrm{HC}}^{*}(A, B)$ et $\widehat{\mathrm{HC}}(B, A)$ de la multiplication nulle. Alors les endomorphismes $\left(L \psi_{A}^{l}\right)$, munissent $\widehat{\mathrm{HC}}^{*}(A, B)$ (resp. $\left.\widehat{\mathrm{HC}}^{*}(B, A)\right)$ d'une structure de $\gamma$-anneau.

On termine ce paragraphe par quelques considérations sur les $\gamma$-filtrations associées à ces $\gamma$-anneaux. On conserve les notations précédentes.

Les opérations $\left(L I \psi_{A}^{l}\right)_{l}\left(\right.$ resp. $\left.\left(R I \psi_{A}^{l}\right)_{l}\right)$ sur $\mathrm{HH}^{*}(A, B)\left(\right.$ resp. sur $\left.\mathrm{HH}^{*}(B, A)\right)$ induisent des opérations $\left.\left(L I \gamma_{A}^{l}\right)_{l}\right)$ (resp $\left.\left(R I \gamma_{A}^{l}\right)_{l}\right)$ sur $\mathrm{HH}^{*}(A, B)$ (resp. sur $\mathrm{HH}^{*}(B, A)$ ), d'où une $L I \gamma$-filtration (resp. $R I \gamma$-filtration). Le iième terme de la filtration $F_{L I \gamma}^{i}=F_{L I \gamma}^{i} \mathrm{HH}^{*}(A, B)\left(\right.$ resp. $\left.F_{R I \gamma}^{i}=F_{R I \gamma}^{i} \mathrm{HH}^{*}(B, A)\right)$ est le sous-module de $\operatorname{HH}^{*}(A, B)$ (resp. de $\left.H H^{*}(B, A)\right)$ engendré par les éléments

$$
L I \gamma_{A}^{l}(\alpha)\left(\operatorname{resp} . R I \gamma_{A}^{l}(\beta)\right) \text { pour } l \geqq i .
$$

On vérifie aisément que $F_{L I \gamma}^{1}=\operatorname{HH}^{*}(A, B)$ et $F_{R I \gamma}^{1}=\operatorname{HH}^{*}(B, A)$. Cependant, contrairement au cas de l'homologie cyclique, le deuxième terme de la filtration est, en général, strictement contenu dans le premier terme. Cela provient du fait que $\gamma^{1}$ est combinaison linéaire de $\gamma^{2}, \ldots, \gamma^{l}, \ldots$ en degré $n$ fixé, mais non pour tous les degrés simultanément.

De façon analogue, on déduit des $\gamma$-opérations et une $\gamma$-filtration sur la cohomologie cyclique bivariante $\widehat{\mathrm{HC}}^{*}$. On vérifie qu'il n'y a même plus égalité, en général, entre le premier terme de la filtration et $\widehat{\mathrm{HC}}^{*}$.

\section{RÉFÉRENCES}

1. M. Atiyah, D. O. Tall, Group representations, $\lambda$-rings and the $\lambda$-homomorphisms, Topology 8 (1969), 253-297.

2. D. Burghelea, M. Vigué-Poirrier, Cyclic homology of commutative algebras I, Lecture Notes in Math. 1318 Springer, Berlin-Heidelberg-New York (1988), 51-72.

3. A. Connes, Non-commutative differential geometry, Publ. Math. IHES 62 (1985), 41-144.

4. B. L. Feigin, B. L. Tsygan, Additive K-theory, Lecture Notes in Math. 1289 Springer, Berlin-Heidelberg-New York (1987), 97-209.

5. M. Gerstenhaber, S. D. Schack, A Hodge-type decomposition for commutative algebra cohomology, J. Pure Appl. Algebra 48 (1987), 229-247.

6. T. G. Goodwillie, Cyclic homology, derivations and the free loopspace, Topology 24 (1985), 187-215.

7. T. G. Goodwillie, Relative algebraic K-theory and cyclic homology, Ann. of Math. 124 (1986), $347-402$. 
8. D. K. Harrison, Commutative algebras and cohomology, Trans. Amer. Math. Soc. 104 (1962), 191-204.

9. J. D. S. Jones, C. Kassel, Bivariant cyclic theory, K-theory 3 (1989), 339-365.

10. C. Kassel, Cyclic homology, comodules and mixed complexes, J. Algebra 107 (1987) 195-216.

11. C. Kassel, K-théory algébrique et cohomologie cyclique bivariantes, C.R.Acad.Sc. Paris 306 (1988), 799-802.

12. C. Kassel, Caractère de Chern bivariant, K-theory 3 (1989), 367-400.

13. C. Kassel, Homologie cyclique, caractère de Chern et lemme de perturbation, J. Reine Angew. Math. 408 (1990), 159-180.

14. C. Kassel, Une formule de Künneth pour la décomposition de l'homologie cyclique des algèbres commutatives, Math. Scand. 27-33.

15.J. L. Loday, Opérations sur Thomologie cyclique des algèbres commutatives, Invent. Math. 96 (1989), 205-230.

16. J. L. Loday, D. Quillen, Cyclic homology and the Lie algebra homology of matrices, Comment. Math. Helv. 59 (1984), 565-591.

17. T. Natsume, S. D. Schack, $A$ decomposition for the cyclic cohomology of a commutative algebra. J. Pure Appl. Algebra 61 (1989), 273-282.

18. D. Quillen, On the (co)homology of commutative rings, Proc. Sympos. Pure Math. 17 AMS(1970), 65-87.

19. M. Vigué-Poirrier, Cyclic homology and Quillen homology of a commutative algebra, Lecture Notes in Math. 1318 Springer, Berlin-Heidelberg-New York (1988), 238-245.

20. M. Vigué-Poirrier, Décompositions de Thomologie cyclique des algèbres différentielles graduées commutatives, K-theory 4 (1991), 399-410.

INSTITUT DE RECHERCHE MATHÉMATIQUE AVANCÉ

C.N.R.S.-UNIVERSITE LOUIS PASTEUR

7, RUE RENÉ DESCARTES

67084 STRASBOURG CEDEX

FRANCE
UNIVERSITETET I OSLO MATEMATISK INSTITUTT POSTBOKS 1053 BLINDERN 0316 OSLO 3 NORGE 